\title{
Blunt Splenic Injury: Efficacy of Superselective Splenic Artery Embolization
}

\section{Chou $\mathrm{CP}^{1,2}$, Kim $\mathrm{YH}^{2}$, Tresoldi $S^{2,3}$, Tikh $\mathrm{EI}^{2}$, Baker $\mathrm{S}^{4}$, Kandarpa $\mathrm{K}^{2}, \mathrm{Kim}^{5}$}

${ }^{1}$ Department of Radiology, Kaohsiung Veterans General Hospital, Kaohsiung 813, Taiwan, ${ }^{2}$ Department of Radiology, UMass Memorial Medical Center, University of Massachusetts Medical School, Worcester, MA, 01655 USA, ${ }^{3}$ Diagnostic and Interventional Radiology Department, A.O. San Paolo Via A. di Rudini 8, 20142 Milan, Italy, ${ }^{4}$ Department of Quantitative Health Sciences, University of Massachusetts Medical School, Worcester, MA, 01655 USA, ${ }^{5}$ Department of Radiology, Boston Medical Center, Boston University, Boston, MA 02118 USA.

\begin{abstract}
Objective: To evaluate the efficacy of superselective splenic artery embolization (SAE) using a coaxial catheter technique in patients with blunt splenic injury. Patient Selection and Methods: We retrospectively reviewed cases of 24 consecutive patients undergoing splenic angiography for blunt splenic injury at a Level 1 trauma center. After angiographic confirmation of splenic injury, superselective SAE was performed using gelfoam pledgets $(n=15)$, with or without coils, and liquid embolic materials $(n=9)$. All procedures were performed through a microcatheter advanced coaxially through a selective angiographic catheter. Severity of splenic injury was graded using CT imaging. The angiographic findings were retrospectively divided into four groups. Outcome measures included technical success, complications and recurrence of symptoms requiring additional intervention or surgery despite embolization. Results: All cases were technically successful, with immediate occlusion of targeted vessels after embolization. One patient underwent distal pancreatectomy- splenectomy 3 days after splenic embolization due to symptoms related to distal pancreatic injury, unrelated to the angiographic intervention. A second embolization was performed 1-3 days after initial embolization in 4 patients with clinical suspicion of rebleeding. One of these four patients underwent splenectomy 3 days after the second embolization, during exploration for associated bowel and diaphragmatic injury. A second patient underwent splenectomy 1 day after a second embolization due to need for continued transfusions. The splenic salvage rate was not significantly related to $\mathrm{CT}$ grade of splenic injury ( $\mathrm{p}=1.0)$ or angiographic classification $(\mathrm{p}=0.8)$. Conclusion: Superselective SAE can be performed as a safe alternative to splenectomy in patients with blunt splenic trauma, particularly when there is no additional major organ injury.
\end{abstract}

Keywords: Embolization, Nonsurgical management, Splenic angiography, Splenic injury, Splenic laceration.

Correspondence to: Dr Young H Kim, MD Department of Radiology, UMass Memorial
Medical Center, University of Massachusetts Medical School, Worcester, MA, 01655 USA

Email: kimy@ummhc.org 


\section{Introduction}

The spleen is the most commonly injured organ in patients with abdominal trauma. CT imaging has made the diagnosis of splenic injury increasingly accurate. In the early 1990s, splenectomy was the treatment of choice for splenic rupture. Due to susceptibility to infections, including overwhelming sepsis, that can occur post splenectomy with an associated high mortality rate, efforts are made to preserve the spleen using various surgical and nonsurgical approaches. ${ }^{1}$

Splenic artery embolization (SAE) was first introduced in 1981 by Sclafani et al. for management of splenic injuries. ${ }^{2}$ Recently, SAE has been used as an adjunct to nonoperative management of the injured spleen. ${ }^{3}$ Several studies over the last decade documented that nonoperative management using proximal SAE improves splenic salvage rates in patients found to have angiographic abnormalities. Distal or superselective SAE was then introduced for selected cases in more recent studies, since new microcatheters were being widely used and there was concern that proximal SAE could unnecessarily compromise parenchymal perfusion of the entire spleen. With superselective embolization of bleeding segmental arteries, the remaining splenic parenchyma has an improved chance of survival and maintaining functionality. ${ }^{4}$ It is important to be aware of the therapeutic effect and clinical outcomes of superselective SAE as an adjunct to nonsurgical management for splenic injury. We retrospectively analyzed the technical success, complications, and recurrence of symptoms requiring additional intervention or surgery after superselective SAE.

\section{Patient Selection and Methods}

We searched the angiogram registry of our American College of Surgeons accredited Level 1 trauma center for patients with blunt splenic injury who were treated with superselective SAE during a 5-year period. Superselective SAE was defined as embolization involving distal to first terminal splenic arterial branches. Twenty-four patients were identified and included in this study. After obtaining hospital IRB approval, retrospective review of all twenty-four patients' medical records and radiologic images was performed. Findings at angiography leading to embolization, materials used for embolization, and angiographic results were recorded.

All patients with blunt abdominal trauma were initially assessed and resuscitated in the emergency department according to the Advanced Trauma Life Support (ATLS) guidelines. Each patient underwent a contrast-enhanced abdominal CT scan on the day of injury. Severity of splenic injury was graded using CT imaging (Grade $\mathrm{I}$ to $\mathrm{V}$ according to laceration severity and hematoma) following the classification of the American Association for the Surgery of Trauma (AAST). ${ }^{5}$ Hemoperitoneum observed on CT images was recorded as focal (limited to the perisplenic space) or significant (intra-abdominal blood in addition to the perisplenic space).

Angiography was ordered at the discretion of the admitting trauma surgeon and performed by interventional radiologists in a dedicated angiograhy suite. Twenty-two patients (92\%) underwent angiography within one day after injury. An additional two patients (8\%) underwent angiography on day 3 following 
injury. Angiography was performed via femoral arterial access in either groin. After placing a $5 \mathrm{Fr}$ vascular sheath (Terumo, Tokyo, Japan) and a multi-sidehole 4-5 Fr pigtail catheter (Cordis, Miami, FL, USA), an abdominal aortogram was initially performed in most cases for evaluation of vascular anatomy and pathologies associated with the trauma. A 4 or 5 Fr catheter (Cobra Glidecath, Terumo, Tokyo, Japan) was then placed into the mid splenic artery. After selective cannulation of the splenic artery, a splenic arteriogram was obtained. The angiographic findings were retrospectively divided into four groups according to the modified classification by Liu et al. ${ }^{3}$

Group 1: extravasation of contrast media extending beyond the splenic parenchyma, Group 2: extravasation within the splenic parenchyma,

Group 3: abnormal disruption of terminal arteries without extravasation, and

Group 4: variable degree of avascularity and irregularity in accumulation of contrast media

The superselective SAE technique used a 3Fr microcatheter (Renegade; Boston Scientific, Natick, MA), which was advanced coaxially through a 4 or $5-\mathrm{Fr}$ angiographic catheter. After angiographic confirmation of injured branches and subsequent superselective catheterization, embolizations were carried out under fluoroscopy with injection of solid or liquid embolic agents mixed with contrast media. A completion splenic arteriogram was performed to confirm successful occlusion of appropriate vessels and to assess for any interventional complication.
Seven patients underwent repeat abdominal CT imaging 1-7 days after superselective SAE. Outcome variables were reviewed according to surgical records and discharge notes. Splenic salvage was defined as spleen in situ when the patient was discharged from the hospital. Nonoperative management failure was defined as the need for abdominal exploration regardless of the indication. Complications after SAE were classified as major or minor. Major complications were defined as adverse events believed to be directly related to SAE and which could potentially result in severe disability or death. Minor complications were defined as adverse events not deemed life threatening. Significant splenic infarct was categorized as a major complication and was defined as devascularization of $>25 \%$ of the parenchyma on postprocedural abdominal CT scan. All patients were followed up in our outpatient clinic over a period of 1 to 41 months (mean 12.3 months).

\section{Data Analysis}

The predictive value of $\mathrm{CT}$ grading and angiography classification for spleen salvage was evaluated using the receiver operating characteristic (ROC) methodology. The predictive accuracy was quantified by using the area under the ROC curve (AUC-ROC). Ninety-five percent confidence intervals (95\% CIs) were constructed for the AUCROC. A confidence interval not including 0.5 , was considered to be of significant predictive value. In addition, the distributions of CT grade and angiographic classification in patients having successful proximal SAE were compared with the distributions of patients undergoing splenectomy using the Mann-Whitney U- 


\begin{tabular}{|l|l|l|l|l|}
\hline \multicolumn{5}{|l|}{ Table. 1: Distribution of blunt splenic trauma (by AAST grade) and clinical outcome } \\
\hline Findings & \multicolumn{4}{|c|}{ Splenic Injury CT Grading (AAST) } \\
\hline & II (n=2) & III (n=7) & IV (n=12) & V (n=3) \\
\hline $\begin{array}{l}\text { Significant } \\
\text { hemoperitoneum on CT }\end{array}$ & $1(50 \%)$ & $5(71 \%)$ & $11(92 \%)$ & $3(100 \%)$ \\
\hline $\begin{array}{l}\text { Contrast medium } \\
\text { extravasation on CT }\end{array}$ & $0(0 \%)$ & $5(71 \%)$ & $7(58 \%)$ & $1(33 \%)$ \\
\hline $\begin{array}{l}\text { Need a second } \\
\text { splenic embolization }\end{array}$ & $0(0 \%)$ & $0(0 \%)$ & $4(36 \%)$ & $0(0 \%)$ \\
\hline Spleen salvage & $2(100 \%)$ & $7(100 \%)$ & $10(83 \%)$ & $2(67 \%)$ \\
\hline
\end{tabular}

\begin{tabular}{|c|c|c|c|c|}
\hline $\begin{array}{l}\text { Angiographic } \\
\text { finding }\end{array}$ & $\begin{array}{l}\text { Group 1: } \\
\text { Extravasation } \\
\text { beyond the } \\
\text { spleen }(n=2)\end{array}$ & $\begin{array}{l}\text { Group } 2 \text { : } \\
\text { Extravasation } \\
\text { within the } \\
\text { spleen }(n=14)\end{array}$ & $\begin{array}{l}\text { Group } 3 \text { : Abnormal } \\
\text { disruption of terminal } \\
\text { arteries without } \\
\text { extravasation }(n=4)\end{array}$ & $\begin{array}{l}\text { Group } 4 \text { : A variable } \\
\text { splenic avascularity } \\
\text { and irregularity in } \\
\text { accumulation of } \\
\text { contrast media }(n=4)\end{array}$ \\
\hline $\begin{array}{l}\text { Splenic } \\
\text { salvage }\end{array}$ & $1(50 \%)$ & $12(86 \%)$ & $4(100 \%)$ & $4(100 \%)$ \\
\hline $\begin{array}{l}\text { Second } \\
\text { Embolization } \\
\text { required }\end{array}$ & $1(50 \%)$ & $3(21 \%)$ & $0(0 \%)$ & $0(0 \%)$ \\
\hline
\end{tabular}

test. Differences associated with p-values $\leq 0.05$ were considered statistically significant. All computations were performed using the SPSS Version 14 statistical software (SPSS Inc. Chicago, IL).

\section{Results}

The study population consisted of 15 males and 9 females, with a mean age of 31 years (age range, 14-71 years). According to the AAST splenic injury CT grading scale, no patients had Grade I splenic injury, 2 patients (8\%) had Grade II, 7 (29\%) had Grade III, 12 (50\%) had Grade IV, and 3 (13\%) had Grade $\mathrm{V}$ splenic injury (Table. 1). The mean injury grade by CT was 3.7. On CT imaging, active extravasation of contrast medium was noted in $13(54 \%)$ and significant hemoperitoneum in $20(83 \%)$ out of 24 patients. Four $(16 \%)$ of the 24 patients, all of whom had CT Grade IV splenic injury, required repeat SAE.

Fifteen of 24 patients were treated with gelfoam slurry $(\mathrm{n}=13)$, stainless steel microcoils (VortX, Target Vascular, Boston Scientific Corp., Cork, Ireland) $(\mathrm{n}=1)$, or combinations of both $(n=1)$. Nine of 24 patients were treated with a collagen and thrombin compound (D-Stat Flowable Hemostat; Vascular Solutions Inc, Minneapolis, USA), which was approved by the hospital IRB for use in SAE. The total amount of D-Stat used ranged from 0.1 to $0.6 \mathrm{~mL}$ (mean $0.28 \mathrm{~mL}$ ).

The findings on angiography were divided into four groups as mentioned above and further detailed in Table 2. Contrast media 


\begin{tabular}{|l|l|l|}
\hline \multicolumn{3}{|l|}{ Table. 3: Complications of superselective SAE and clinical outcome } \\
\hline Complication & No. & Splenic Salvage \\
\hline Major & 2 & 1 \\
\hline Delayed diagnosis of pancreatic trauma & 1 & 0 \\
\hline Delayed splenic rupture & 1 & 1 \\
\hline Minor & 5 & 5 \\
\hline Significant infarct & 2 & 2 \\
\hline Non-target area infarct & 2 & 2 \\
\hline Coil migration & 1 & 1 \\
\hline
\end{tabular}

extravasation or blush at angiography was noted in $67 \%(16 / 24)$ of patients. Patients in angiographic Group 1 (50\%) and Group 2 $(25 \%)$ had a greater rate of repeated embolization compared with Group $3(0 \%)$ and Group $4(0 \%)$. On selective splenic arteriography, sixteen $(66 \%)$ of 24 patients had extravasation of contrast media within or extending beyond the splenic parenchyma.

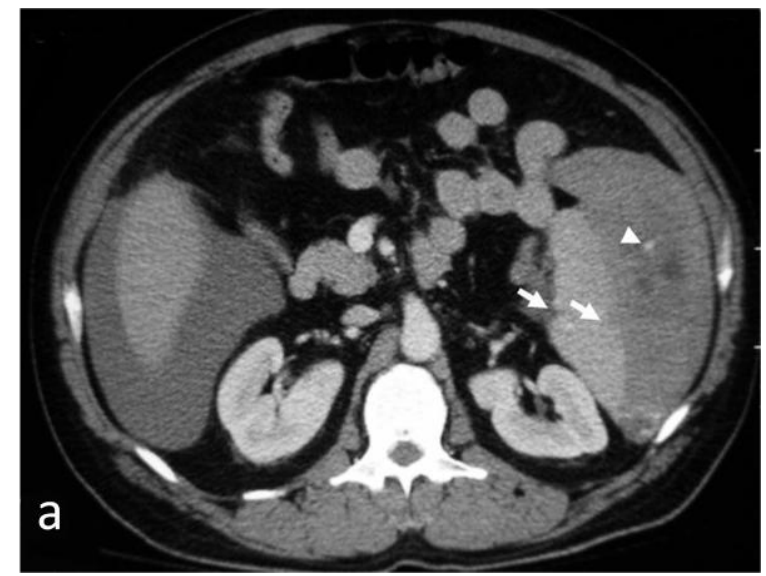

Fig. 1: Images obtained in a 25-year-old man with grade III splenic injury from motor vehicle accident. (a) Axial contrastenhanced CT scan shows splenic parenchymal laceration (arrows) with active extravasation of contrast material (arrowhead). Intraperitoneal hemorrhage is seen adjacent to the liver.

Every patient demonstrated successful embolization of injured vessels on postembolization splenic arteriogram (Fig. 1). One patient underwent surgical exploration three days after superselective SAE because of persistent back and abdominal pain Pancreatic tail laceration that was not noticed at CT was found during emergent laparotomy. This patient underwent distal pancreatectomy and splenectomy due to delayed diagnosis of pancreatic tail injury.

Four $(16 \%)$ out of the twenty-four superselective SAE patients underwent a second angiography, using superselective $(n=3)$ or proximal $(n=1)$ SAE, due to recurrent clinical signs and symptoms of continuous splenic hemorrhage. Findings in these patients included contrast extravasation $(n=1)$ or vessel recanalization $(n=3)$. Of these four patients, one patient underwent splenectomy 3 days after the second embolization due to concern of bowel and diaphragmatic injury associated with the initial abdominal blunt trauma. Another patient underwent splenectomy one day after the second embolization because of the need for continuous blood transfusions.

Splenic salvage occurred in 21 out of 24 patients $(87 \%)$. Patients who needed repeat SAE demonstrated a decreased rate $(50 \%)$ of splenic salvage. There was no immediate mortality related to either splenic injury or superselective SAE treatment. However, one delayed mortality occurred three weeks post superselective SAE, attributed to initial multiple trauma complicated by sepsis. 
Procedure related complication is sumarized in Table 3. Major complications occurred in two patients. One patient presenting with persistent abdominal pain had a delayed diagnosis of distal pancreatic injury, unrelated to the angiographic intervention, and was found during subsequent surgery. Another patient had delayed rupture of splenic parenchyma in the region of SAE, which was observed on follow-up CT scan. This patient had minimal clinical symptoms and was treated conservatively.

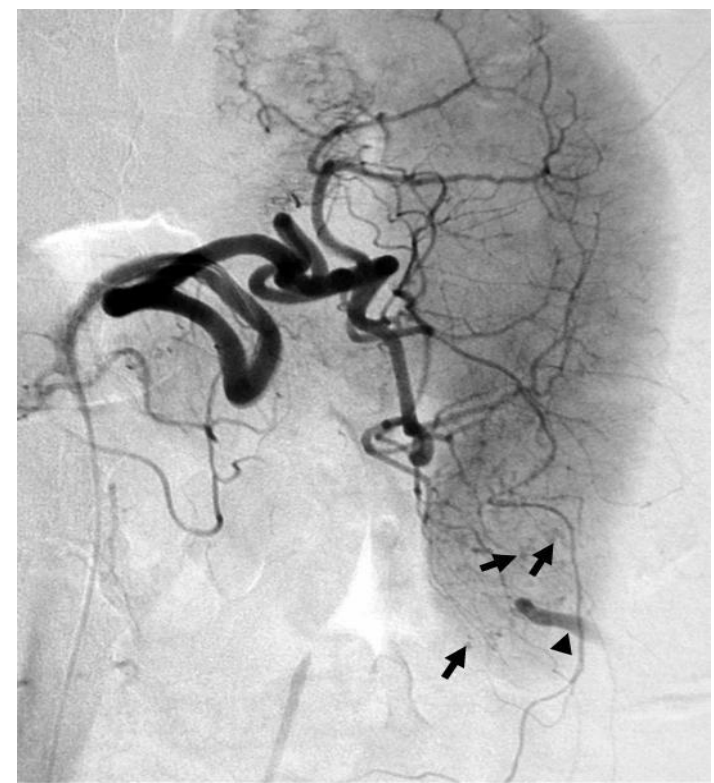

Fig. 1b: Anteroposterior selective splenic arteriogram shows foci of extravasation within the splenic parenchyma (arrows) and beyond the parenchyma (arrowhead) in the lower pole of the spleen.

Two patients had a peripheral infarct in a non-target territory of the splenic parenchyma. We did not routinely use a balloon catheter during embolization to prevent reflux of embolic agents. The infarcted regions were usually limited and peripheral in distribution, and did not cause secondary clinical consequences. In one patient, a single coil migration to a peripheral branch was noted and additional gelfoam pledgets were used to occlude the vessels. Four patients experienced an episode of fever during hospitalization, but the source of fever was attributed to other wounds or pneumonia. After SAE, follow-up CT showed tiny foci of gas in the splenic parenchyma on two asymptomatic patients. Minimal amount of gas observed on imaging in the splenic parenchyma, which is not an uncommon CT finding after embolization, was not considered to be a complication in this study.

The area under the ROC curve was 0.508 (95\% CIs 0.211-0.804) for CT grading and 0.556 (95\% CIs $0.242-0.869$ ) for angiography classification (Fig. 2). Neither CT grade nor angiographic classification demonstrated significantly predictive value for splenic salvage. The distributions of CT grade $(\mathrm{p}=1.0)$ and angiographic classification $(\mathrm{p}=0.8)$ had no significant difference between patients with splenic salvage vs. splenectomy.

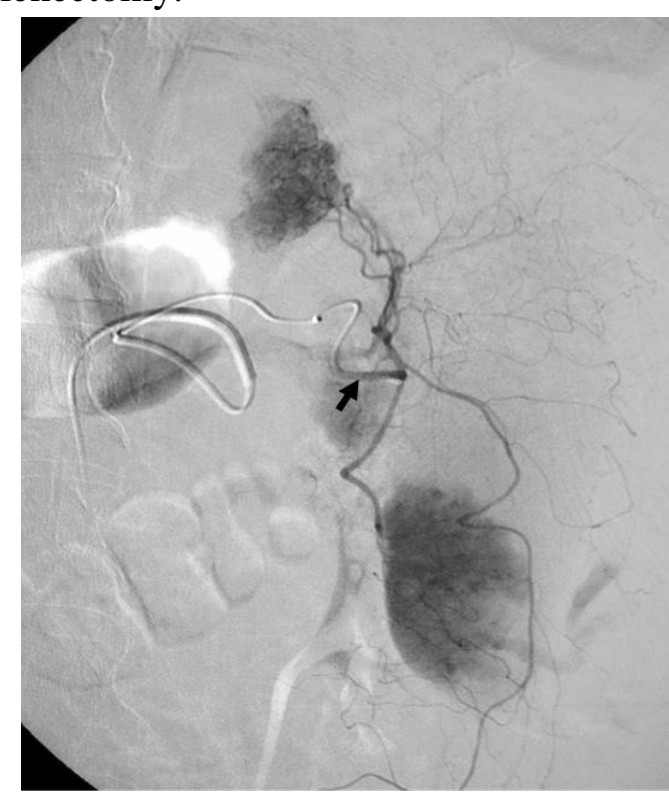

Fig. 1c: The superselective splenic arteriogram is obtained using a 3-Fr microcatheter (arrow) and splenic artery is embolized by Gelgoam pledgets in superselective branches. 


\section{Discussion}

Splenic injury is common in patients with blunt abdominal trauma. Many patients that present with multisystem trauma have clinical findings of hypotension and tachycardia, and they undergo CT imaging for evaluation of potential injuries. Abdominal CT imaging is widely used in the assessment of patients with abdominal trauma. It is a reliable study for the evaluation of the presence and extent of hemoperitoneum, grading of splenic injury, and exclusion of associated injuries that may require surgery. ${ }^{6} \mathrm{CT}$ imaging, however, does have limitations. It often does not demonstrate whether the source of bleeding is arterial, venous, or parenchymal in nature. Some reports in the literature have demonstrated that CT based splenic injury grading and presence of extravasation of contrast medium is not reliable in the management of splenic injury. ${ }^{7,8}$

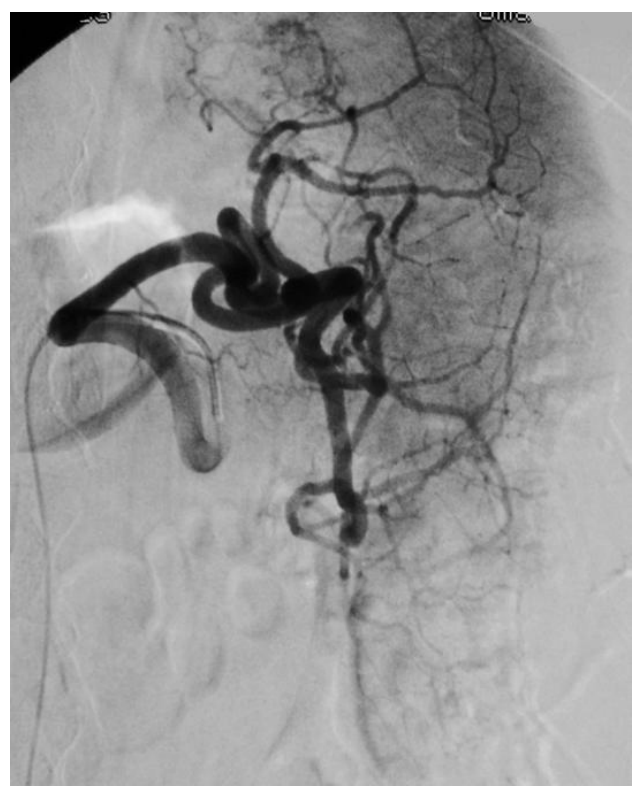

Fig. 1d: Anteroposterior selective splenic arteriogram obtained after superselective SAE with Gelfoam pledgets shows complete occlusion of injured vessels. The remaining splenic vessels are patent.
In the past, the standard treatment for splenic injury was splenectomy. The risk of lifelong susceptibility to infectious complications after splenectomy, particularly a rare but highly fatal syndrome of overwhelming postsplenectomy sepsis in adult patients, was the major impetus for the development of splenic salvage procedures. ${ }^{1}$ The surgical methods proposed for splenic salvage and preservation of important splenic function are splenorrhaphy, partial splenectomy ${ }^{9}$ and autotransplantation. ${ }^{10}$ In addition to the development of surgical splenic preservation techniques, nonsurgical approaches using SAE to treat splenic injury have been developed for both pediatric and adult patients with success rates of up to $90 \% .{ }^{11} \mathrm{~A}$ multi-institutional study from the Eastern Association for the Surgery of Trauma (EAST) demonstrated during a five-year period (1993-1997) that direct splenectomy rates for blunt splenic injury dropped from a mean of $52 \%$ to $39 \%$ due to greater numbers of patients undergoing non-operative management. ${ }^{12}$ Two studies published after 2000 report a rate of direct operative intervention as low as 23 to $27 \% .^{13,14}$ The current recommendation for hemodynamically stable adult and pediatric patients with splenic injuries is nonoperative management, if they have no additional injuries requiring laparotomy. ${ }^{6}$ Recent studies enabled the development of guidelines that can be used to select patients appropriate for nonsurgical therapy, and subsequently reduce the incidence of splenectomy and its associated complications. ${ }^{15}$

Angiography can be used for the detection of active splenic bleeding. Depending on the severity of injury, lack of extravasation of contrast medium on a splenic angiogram has 
been shown to be a predictor of successful nonoperative management with 90-100\% certainty. ${ }^{16,17}$ Since a significant portion $(10 \%)$ of patients without contrast extravasation still required repeat angiography or even surgery, SAE is performed not only in patients with active bleeding on angiography, but also in patients with disruption of terminal splenic arteries on angiography and significant hemoperitoneum on CT imaging. ${ }^{18}$

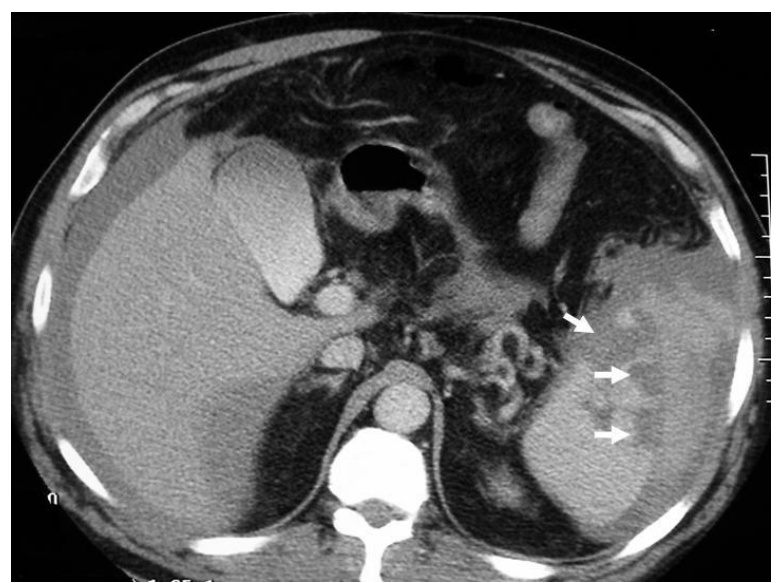

Fig. 1e: On day 7 after superselective SAE, axial contrast-enhanced CT scan shows small peripheral splenic infarcts (arrows) in the lower pole.

Splenic angiography with embolization for hemostasis has been described as an effective tool that increases the success rate in patients undergoing nonoperative treatment of splenic injury. ${ }^{18}$ In 1981, Sclafani introduced SAE for occlusion of the proximal splenic artery in cases of splenic injury. He also reported a second larger SAE series in which the majority of splenic injuries could be managed nonoperatively. ${ }^{17}$ There are various ways to perform SAE, including proximal or distal embolization or a combination of both. Initially, proximal SAE was preferred because it was easily preformed and offered the same effect as surgical ligation. ${ }^{2-17}$ Hagiwara et al. described the advantage of proximal SAE in patients with less severe splenic injuries and emergent active bleeding or technically difficult cases. ${ }^{18}$ Bessoud et al. discussed how proximal splenic artery embolization using coils significantly reduces the intrasplenic blood pressure, thereby facilitating thrombosis formation at bleeding sites and preserving splenic function. ${ }^{19}$ After main splenic artery embolization, blood flow to the spleen could be supplied through rich collateral circulation from the left gastric and gastroepiploic arteries. An understanding of collateral flow pathways to previously embolized or injured areas is essential for planning a safe and effective SAE. However, studies have shown that nearly 20-29\% of patients treated with main splenic artery coil embolization have significant $(>25 \%$ parenchyma) subsequent infarction. ${ }^{20,21}$ Concerns about splenic function after proximal SAE remain in question.

A proximal or distal SAE or a combination of both can be performed for the treatment of splenic injury. Superselective SAE has the potential benefit of providing hemostasis to injured vessels and preserving perfusion to the remainder of the splenic parenchyma. This technique involves placing a microcatheter $(<3 \mathrm{Fr}$ ) coaxially via a 4-5 Fr catheter directly adjacent to documented intraparenchymal vascular injuries at the time of angiogram. Superselective SAE can then be performed with solid or liquid embolic agents. Potential complications of superselective SAE include the possibility of rebleeding, focal infarction, and vascular injury. ${ }^{18}$

Our study demonstrates that superselective SAE can be performed successfully in patients with high-grade splenic injury. The 
average AAST injury grade of the splenic injuries in this series is 3.7. Grade 4 splenic injury was the most common observed, occurring in $50 \%$ of the patients. Nearly $63 \%$ of the patients had one or more foci of contrast extravasation at the time of angiography. This is comparatively much greater than that found in other series. Despite significant injury severity, splenic salvage in our patients selected for superselective SAE was $87 \%$.

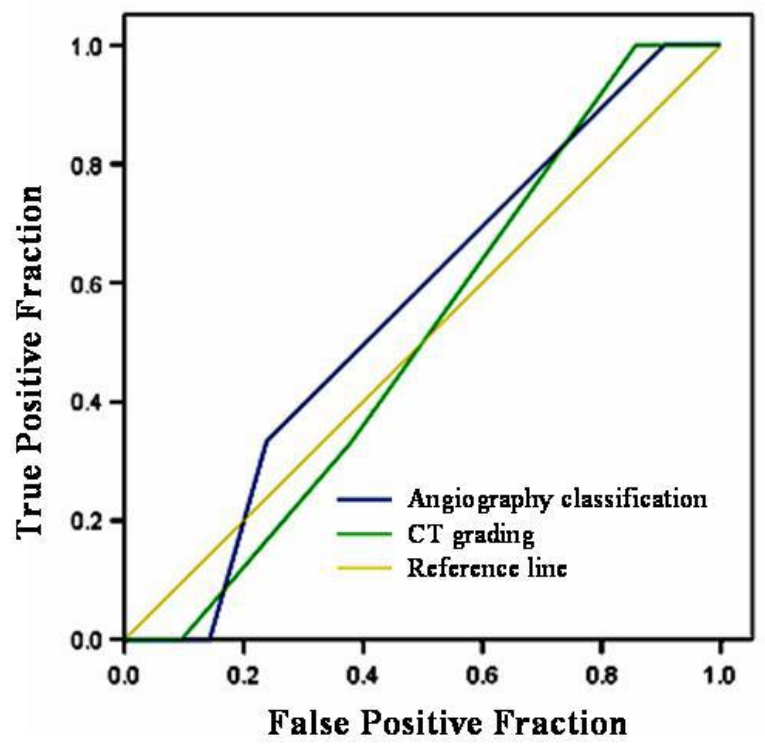

Fig. 2: The predictive value of CT grading and angiography classification for spleen salvage was evaluated using the area under the ROC curve. Both CT grading and angiographic classification demonstrated no predictive value for splenic salvage or splenectomy.

In grading splenic parenchymal and vascular injuries, we used the established practices of CT and angiographic classification. ${ }^{3,5}$ None of our patients with lower grade splenic injury (Grade II and III) or lack of contrast medium extravasation at angiography (Group 3 and 4) underwent splenectomy. However, both CT grading and angiography classification failed to predict outcome of superselective SAE. Our data also demonstrated that neither CT grading or angiography classification showed statistically significant differences in patients with splenic salvage vs. splenectomy. Four patients developed complications related to splenic infarction. Two patients demonstrated significant infarcts, while two other patients had non-target area infarct in the spleen. Splenic infarcts were usually managed conservatively. None of the patients with partial splenic infarct developed subsequent problems in our series. Prophylactic antibiotic administration was not recommended in patients after SAE for traumatic injury. ${ }^{17}$ We did not routinely use antibiotics to prevent infection following embolization. Four patients experienced an episode of fever during hospitalization due to infected soft tissue wounds or pneumonia. In our study, no patient was suspected of having fever related to embolization. Splenic abscess is an uncommon complication following non-operative management of splenic trauma. ${ }^{22}$ Splenic abscess formation requires treatment with antibiotics in combination with percutaneous drainage or even splenectomy. No splenic abscess was noted in our study. Limited amount of gas in the splenic parenchyma following SAE may be a frequent occurrence after embolizations, and in asymptomatic patients, observation is usually appropriate for tiny gas collections. ${ }^{23}$ In our series, two of the patients had tiny foci of gas in the spleen after SAE and they were followed up clinically with no further management necessary.

Patients with multiple trauma may present with recurrent hypotension after initial treatment, but the causes are usually multiple and difficult to distinguish. Under such circumstances, the trauma surgeon should evaluate the patient carefully and decide 
whether nonsurgical management of the splenic injury is appropriate. Although for hemodynamically unstable patients, there is currently not a clear consensus regarding indication of surgery vs SAE, clinically unstable patients usually undergo surgery to enhance patient's safety. Ability to determine splenic immune function after partial splenectomy or SAE is still limited and controversial. ${ }^{24} \mathrm{~A}$ study of traumatic patients showed that partial splenectomy and splenorrhaphy resulted in normal splenic reticuloendothelial function that was indistinguishable from intact spleens. ${ }^{25} \mathrm{We}$ believe that proximal SAE may compromise splenic immune function since the main splenic arterial blood flow is substantially reduced. Furthermore, collateral splenic vessels can be responsible for failure of proximal $\mathrm{SAE}$ to allow non-operative management. Bleeding through collateral vessels can be persistent and decrease the hemostatic effect of proximal embolization. ${ }^{18}$ On the other hand, paucity of collateral vessels can cause significant post procedure infarction of the spleen. With superselective SAE of a bleeding segmental artery, there is an improved chance of maintaining the immune function of the spleen. Superselective SAE is the procedure preferred to proximal SAE whenever feasible in our hospital. We believe that superselective SAE maintains the splenic immune function and achieves hemostasis more effectively than proximal SAE.

The main limitations of this study are that it is retrospective and that there was lack of a prospective protocol for angiography and SAE. Like most trauma centers, our institution trauma surgeons manage patients considering hemodynamic stability, need for transfusions, CT grading and angiographic findings. In this series, not all patients underwent repeat CT imaging after SAE. Among patients that underwent repeated CT, the time interval between SAE and the second CT imaging was not constant. Therefore, accurate overall prevalence of splenic infarcts after SAE cannot be determined. With relative short term CT follow-up, the exact area of infarct could be overestimated. In another study, splenic infarct after SAE was shown to resolve without sequela in the majority of patients. ${ }^{21}$

Radiologists usually perform SAE depending on CT grading of the splenic injury, presence of active extravasation and the hemodynamic condition of the patient. New generation multi-slice helical CT imaging may provide better correlation between splenic injury and operative findings. ${ }^{26}$ Unfortunately, hemoperitoneum detected on CT may originate from other arterial branches and veins in addition to the splenic arteries. Subtle abdominal visceral injuries could be missed on CT imaging or during early clinical evaluation. For example, one patient in our series had pancreatic injury that was not detected on CT and subsequently underwent conservative management with SAE. Ultimately that patient required surgical exploration with distal pancreatectomy and splenectomy. Clinicians that treat trauma patients should be aware of the potential for delayed diagnosis of coexisting pancreatic, diaphragmatic, and bowel injuries in patients undergoing nonoperative management of splenic injuries. Trauma surgeons usually determine the management of splenic injury and also monitor the conditions of patients selected for nonoperative management. Cooperation between interventional radiologists and trauma surgeons is important for appropriate 
management and timely treatment of splenic injuries and improvement in splenic salvage rates.

\section{Conclusion}

Superselective SAE with a microcatheter can be performed as a safe alternative to surgical management in patients with blunt splenic injuries, especially in those that are hemodynamically stable and without additional major organ injury. Effective communication between trauma surgeons and interventional radiologists is imperative when nonoperative management is utilized.

\section{References}

1. Pachter HL, Guth AA, Hofstetter SR, Spencer FC. Changing patterns in the management of splenic trauma: the impact of nonoperative management. Ann Surg 1998;227:708-717.

2. Sclafani S. The role of angiographic hemostasis in salvage of the injured spleen. Radiology. 1981;141:645-650.

3. Liu PP, Lee WC, Cheng YF et al. Use of splenic artery embolization as an adjunct to nonsurgical management of blunt splenic injury. J Trauma. 2004;56:768772.

4. van der Hul RL, van Overhagen $H$, Dallinga RJ, de Graaf PW. Splenic salvage by superselective embolization after blunt abdominal trauma. Eur J Surg 2001;167: 73-75.

5. Moore EE, Cogbill TH, Jurkovich GJ, Shackford SR, Malangoni MA, Champion HR. Organ injury scaling: spleen and liver (1994 revision). J Trauma 1995;38:323-324.

6. Knudson MM, Maull KI. Nonoperative management of solid organ injuries: past, present, and future. Surg Clin North Am 1999;79:1357-1371.

7. Omert L, Salyer D, Dunham C, Porter J, Silva A, Protetch J. Implications of the "contrast blush" finding on computed tomographic scan of the spleen in trauma. J Trauma 2001;51:272-277.

8. Becker CD, Spring P, Glattli A, Schweizer W. Blunt splenic trauma in adults: can CT findings be used to determine the need for surgery? AJR Am J Roentgenol 1994,162:343-347.

9. Tulikoura I, Lassus J, Konttinen YT, Juutilainen T, Santavirta S. A safe surgical technique for the partial resection of the ruptured spleen: a clinical report. Injury 1999,30:693-697.

10. Leemans R, Manson W, Snijder JA, Smit JW, Klasen HJ, The TH, Timens W. Immune response capacity after human splenic autotransplantation: restoration of response to individual pneumococcal vaccine subtypes. Ann Surg 1999; 229:279-285.

11. Haan JM, Bochicchio GV, Kramer N, Scalea TM. Nonoperative management of blunt splenic injury: a 5-year experience. J Trauma 2005;58:492-498.

12. Harbrecht BG, Peitzman AB, Rivera. Contribution of age and gender to outcome of blunt splenic injury in adults: multicenter study of the eastern 
association for the surgery of trauma. J Trauma. 2001;51:887-895.

13. Bee TK, Croce MA, Miller PR, Pritchard FE, Fabian TC. Failures of splenic nonoperative management: is the glass half empty or half full? J Trauma 2001; 50:230-236.

14. Brasel KJ, Weigelt JA, Christians KK, Somberg LB. The value of process measures in evaluating an evidencebased guideline. Surgery 2003;134:605610.

15. Wahl WL, Ahrns KS, Chen S, Hemmila MR, Rowe SA, Arbabi S. Blunt splenic injury: Operation versus angiographic embolization. Surgery 2004;136: 891899.

16. Haan J, Scott J, Boyd-Kranis RL, Ho S, Kramer M, Scalea TM. Admission angiography for blunt splenic injury: advantages and pitfalls. J Trauma 2001; 51:1161-1165.

17. Sclafani SJ, Shaftan GW, Scalea TM, et al. Nonoperative salvage of computed tomography-diagnosed splenic injuries: utilization of angiography for triage and embolization for hemostasis. J Trauma 1995;39:818-825; discussion 826-827.

18. Hagiwara A, Yukioka T, Ohta S, Nitatori T, Matsuda H, Shimazaki S. Nonsurgical management of patients with blunt splenic injury: efficacy of transcatheter arterial embolization. AJR Am J Roentgenol 1997;169:1151-1156.

19. Bessoud B, Denys A. Main splenic artery embolization using coils in blunt splenic injuries : effects on the intrasplenic blood pressure. Eur radiol 2004; 14:1718-1719.

20. Haan J, Biffl W, Knudson M, et al. Splenic embolization revisited: a multicenter review. J Trauma 2004;56:542547.

21. Killeen KL, Shanmuganathan K, BoydKranis R, Scalea TM, Mirvis SE. CT Findings after Embolization for Blunt Splenic Trauma. J Vas Interv Radiol 2001;12:209-214.

22. Cocanour CS, Moore FA, Ware DN, Marvin RG, Clark JM, Duke JH. Delayed complications of nonoperative management of blunt adult splenic trauma. Arch Surg 1998,133:619-624; discussion 624-625.

23. Haan J, Bochicchio G, Kramer M, Scalea T. Air following splenic embolization: infection or incidental finding? Am Surg 2003, 69: 1036-1039; discussion 10391040 .

24. Ziske CG, Muller T. Partial splenectomy: uses of error. Lancet 2002;359:1144.

25. Traub A, Giebink GS, Smith C,et al. Splenic reticuloendothelial function after splenectomy, spleen repair, and spleen autotransplantation. N Eng J Med 1987; 317:1559-1564.

26. Velmahos GC, Chan LS, Kamel E, et al. Nonoperative management of splenic injuries: have we gone too far? Arch Surg 2000;135:674-679; discussion 679681. 